\title{
Are Primary Trauma Care (PTC) courses beneficial in low- and middle- income countries - A systematic review
}

Keywords: PTC; Primary Trauma Care; Trauma; Education; Course

\begin{abstract}
:
Background:

Injuries remain an important public health concern, resulting in considerable annual morbidity and mortality. In low- and middle-income countries (LMICs), the lack of appropriate infrastructure, equipment and skilled personnel compound the burden of injury, leading to higher mortality rates. As Advanced Trauma Life Support (ATLS) courses remain uneconomical and inappropriate in LMICs, the Primary Trauma Care (PTC) course was introduced to provide an alternative that is both sustainable and appropriate to local resources.
\end{abstract}

Methods:

A systematic review was performed in May 2019, utilising MEDLINE, EMBASE, Cochrane Library and Google Scholar. All studies reporting patient related outcomes (mortality and morbidity rates) and course participant related outcomes (knowledge, confidence and skills) in LMICs were included. PRISMA guidelines were adhered to throughout.

Results:

Nine observational studies were identified (Level 3 evidence). Six studies reported improved knowledge in injury management post-PTC course $(p<0.05)$. Two studies reported improvements in confidence $(p<0.05)$ and one on skill attainment $(p<0.0001)$. One study reported a reduction in mortality rates post-PTC course $(p<0.01)$. 
Conclusion:

Departmental, institutional and personal improvements may occur in clinical practice as a result of formal PTC training of trauma team members in LMICs. Further high-quality research is needed to evaluate this course's effects on observed change in clinical practice and patient outcomes. This may require long-term observational and epidemiological studies to assess improvements in morbidity and mortality.

PROSPERO Registration Number: CRD42019133986 


\section{Introduction}

Injuries remain an important public health concern, resulting in considerable annual morbidity and mortality. In 2012, it was postulated by the World Health Organisation (WHO) that there were in excess of five million fatalities globally attributed to injuries ${ }^{1} .90 \%$ of all injury-related deaths occur in low- and middle-income countries (LMICs) ${ }^{1}$, defined by the World Bank as those with a Gross National Income of less than $\$ 3995^{2}$. The cause of this skewed worldwide distribution of injury-related morbidity and mortality is multifactorial and includes mismatches in provision of trained healthcare professionals and a lack of appropriate infrastructure, trauma centers and equipment ${ }^{3,4}$. Of note, injuries from road traffic accidents, interpersonal violence and war are among the leading causes of death in LMICs ${ }^{4}$. For those who survive their injuries, there is an associated high burden of morbidity accounting for an economic cost of $6 \%$ of global years lived with disability 5 .

Various interventions have been proposed to improve trauma care in LMICs. These include, but are not limited to, pre-hospital system establishment, overall system organization, improvements to the availability of specialty care and trauma care training ${ }^{6}$. Although multiple forms of training exist, they are consistent in providing a positive impact on clinical-effectiveness (injury-related morbidity and mortality) and cost-effectiveness ${ }^{6-8}$. The Advanced Trauma Life Support (ATLS) course, designed in 1978, is seen as the gold standard for initial assessment and management of the seriously injured patient ${ }^{9}$. ATLS aims to provide staff in rural settings with a systematic and concise approach for managing acute trauma ${ }^{9}$. There has been good evidence to support the practice of ATLS principles in trauma, showing improvements in participant knowledge and clinical skills ${ }^{10}$. Over the last few decades, there have been significant reductions in mortality rates from trauma in developed countries. This is secondary to improvements in road safety and 
the management of trauma, establishment of trauma networks and widespread training of trauma teams using courses such as ATLS ${ }^{11-14}$.

However, ATLS in its current structure is unsuitable for widespread incorporation in LMICs. This is multifactorial in nature, but relates to the prohibitive cost of the course, necessary training materials and feasibility of establishing regular courses ${ }^{15,16}$. This is also compounded in many LMICs by a lack of appropriate infrastructure, equipment and skilled personnel to effectively manage trauma ${ }^{17}$. For example, in an observational study of hospitals across ten COSECSA countries (College of Surgeons of East, Central and Southern Africa), there was insufficient capacity to manage trauma, such as a paucity of dedicated emergency units and qualified staff ${ }^{17}$. As a result, those who sustain injuries in LMICs continue to face significant barriers to accessing the necessary trauma care 5 .

Aiming to address the aforementioned problems, the Primary Trauma Care (PTC) course was piloted in $1997^{18,19}$. Marking its $20^{\text {th }}$ anniversary in 2017, the official PTC website claims to have trained personnel in over 70 countries ${ }^{18}$. This course adapted the fundamental principles of the primary and secondary survey that was popularised by ATLS, to create a programme that is not only tailored to the resources available in LMICs, but also one that is sustainable and selfperpetuating ${ }^{20}$. To achieve this, a five-day 'cascading model' is used ${ }^{21}$. Potential instructors are identified during the initial two-day course, and are encouraged to attend the following one-day instructor course ${ }^{19,21}$. Once trained, the instructors will then proceed to run a two-day 'provider' course with the help of external instructors, thus completing five consecutive days ${ }^{19}$.

The initial course itself is cost-free for countries that require and request them. The course manual and materials, including those needed to train instructors, are available for free online ${ }^{20}$. 
Education of participants in the principles of PTC is achieved through means of lectures, small group work and hands-on practical and technical skills sessions ${ }^{22}$. Content covered includes initial assessment using primary and secondary survey, basic and advanced airway and other technical skills, and dealing with trauma to the head, chest, abdomen, pelvis, spine and limbs ${ }^{22-24}$. Whilst the courses manuals and materials are available to support instructors, they are encouraged to adapt the teaching according to the level of participant experience and local resources available 22.

In an effort to understand whether PTC courses are beneficial in LMICs, a systematic review was performed. This aimed to assess both the clinical outcomes following course implementation and educational benefit to participants, establishing whether the PTC course is an effective alternative to ATLS in LMICs.

\section{Methods}

A systematic review was performed in May 2019, utilising the preferred reporting items for systematic reports and meta-analyses (PRISMA) statement as an overall guideline for this study. The review was registered in PROSPERO prior to the execution of the search strategy (CRD42019133986).

The search strategy targeted level three and higher evidence, according to the Oxford Centre for Evidence-Based Medicine. Case reports were excluded. The main outcomes assessed the benefits of PTC course, using variables such as knowledge, skills and confidence of participants. Other outcome measures include mortality and morbidity rates of trauma patients after implementation of PTC courses. 
The search strategy was executed in MEDLINE, EMBASE, PubMed, Cochrane library and Google Scholar. These were searched from commencement until May 11th, 2019 using the following search syntax: ("Primary Trauma Care" OR "PTC course*" OR "Trauma Care course*"). The search algorithm is available in the supplementary materials (Appendix 1). All identified titles and abstracts were analysed using screening inclusion criteria (Table 1). As part of a secondary search, the bibliographies of relevant papers matching our criteria were screened to identify any potential additional citations. The searches were independently conducted by two authors (MK and PS) to prevent any selection bias. Once complete, both lists were amalgamated and duplicates removed. Any disputes were discussed with a third author (CL). Only articles in English were considered, ultimately due to the financial burden that may be incurred in translation.

Articles that assessed the effects of the PTC course on trauma management pre- and post-course in a LMIC were included. Hence, those that reported either on trauma courses other than the official PTC course, or on trauma courses adapted from the PTC course, were excluded.

Data were extracted and incorporated onto a spreadsheet. Information recorded included the list of authors and year of publication, the country and institution that the PTC course(s) was/were delivered, the number of participants in each study if provided, and the outcomes from the study. Once extracted, the data were reviewed to ensure accuracy by an independent author. For each included study, assessment of bias was performed using the Quality Assessment for Quantitative Studies, with each paper given a global rating of "Strong", "Fair" or "Poor" (Appendix 2) 25. Data analysis was performed using R software (2019) and meta-analysis performed only if the results were similar in the intervention, population and outcomes across two or more studies.

\section{Results}


Our search syntax yielded 1184 records across four databases (Figure 1 ). The titles and abstracts of these were scrutinised and articles failing to meet the inclusion criteria were excluded. After this screening, twenty-seven full text articles were assessed for eligibility. Of these, 18 were excluded due to failure in meeting our inclusion criteria or reporting appropriate outcome measures, non-English language and duplicate nature. As a result, nine articles were included in this review (Table 2). Six studies focused on knowledge in management of injuries amongst 2,099 participants, pre- and post-PTC course. Two of these also assessed confidence in managing eight trauma scenarios, and one other also assessed skills. One study reported on response and treatment times, and mortality rates. Another study reported on intended and actual change in clinical practice, and one on the performance of participants in trauma situations, pre- and postintervention. A meta-analysis of the included records was not possible due to the overall heterogeneity of the results.

All nine included papers were published between April 2013 and March 2019, and reported various outcome measures based on PTC courses delivered between 2007 and 2017. Participants recruited varied widely across the studies. Participants varied between the included studies, with seven articles reporting results on a mixture of healthcare professionals (doctors, medical students, nurses, nursing students and other allied healthcare professionals) and two articles reporting results specifically from medical students or doctors respectively (Table 2).

Six articles reported on the effects of the PTC course delivered in LMICs, namely on the participants' change in knowledge pre- and post-course. Completed multiple-choice question (MCQ) tests were obtained from 2,099 of a possible 2,146 participants. All six studies demonstrated a statistically significant improvement in participants' knowledge, ranging from $16.7 \%$ to $31.7 \%$, following attendance at the PTC course $21,23,26-29$ 
Two of these articles enquired about perceived confidence of 1,352 participants in the management of eight trauma scenarios pre- and post-course. Both demonstrated a statistically significant improvement in participants' self-rated confidence post PTC course $(p<0.05)$, with a mean improvement of 19 and $20 \%$ respectively 21,27 .

Only two articles assessed the degree of knowledge and confidence improvement by profession 21,27. Non-doctors were found to show a greater degree of improvement in mean knowledge and confidence scores following the course, when compared to doctors ( 17 and $18 \%$ increase for doctors, versus 20 and $22 \%$ for non-doctors respectively) ${ }^{21,27}$.

In the study conducted by Jawaid et al, assessment of 20 PTC course participants' skills in trauma management was evaluated pre- and post-intervention using a 20-point checklist, following observations of performance during set trauma scenarios ${ }^{28}$. There was a statistically significant improvement in median scores post intervention $(p<0.0001)^{28}$.

One retrospective analysis done by Shi XP et al assessed the impact of PTC training of medical staff on mortality rates at a single medical center $3^{\circ}$. Data were collected from 418,882 trauma cases over a ten-year period and compared these outcomes pre- and post-training. There was no statistically significant difference between the number of trauma cases included in this study before $(159,904)$ and after $(258,978)$ the widespread education of their front-line staff in PTC principles $(p>0.05)^{30}$. This study demonstrated a significant reduction in mortality rates amongst trauma patients, from $31.9 \%$ to $23.1 \%(p<0.01)^{30}$. 
In addition to mortality rate, Shi XP also assessed outcomes of average arrival time to the department and mean time to treatment in their trauma patients ${ }^{30}$. Following PTC training, average rescue time decreased from 16.6 minutes to 15.1 minutes, and average arrival times decreased from 20.8 minutes to 17.4 minutes $(p<0.01)^{30}$.

451 participants from 10 PTC courses in East, Central and Southern African countries were asked to compete a survey to detail intended and actual changes observed in their practice, immediately and six months post-course respectively. This study reported that $92.7 \%$ of their 321 respondents had made changes to their trauma management in practice, notably in the domain of adopting a systematic $A B C D E$ approach ${ }^{22}$. Other benefits reported were moderate improvements in departmental and institutional approaches to trauma management, such as improved teamwork and an increase in staffing levels ${ }^{22}$.

One double-blinded prospective study by Cioè-Peña also examined the impact of the PTC course on clinical practice, however differed in their approach, using an objective outcome measure ${ }^{24}$. Using a checklist scoring system of compliance to primary and secondary survey actions expected during the management of trauma, validated observers reported on adherence to PTC principles. The checklist components included whether there was a pre-hospital alert of patient arrival, performance of primary and secondary surveys, and use of personal protective equipment. CioèPeña reported no change in clinical practice after 148 observed major trauma events, post PTC ${ }^{24}$.

\section{Discussion}

This systematic review aimed to establish whether completion of a PTC course has beneficial effects on trauma management in LMICs. The articles included in this study show that attendance at a PTC course significantly improves candidates' knowledge and confidence when managing 
major trauma. These effects were demonstrated across a wide range of candidates, from doctors and nurses, to non-medically qualified clinical staff. The greatest improvement in knowledge and confidence was seen amongst staff who were not doctors ${ }^{21,27}$. This supports the view that the PTC course is applicable and useful to all health care workers and is of prime importance especially in regions of the world where there is a great shortage of medical personnel. In the literature there is strong evidence linking knowledge to improvements in performance of healthcare staff and patient outcomes ${ }^{31}$. Crucially however, there is a demonstrable attrition of knowledge after 6-12 months ${ }^{26}$, which is similar to reports after ATLS certification ${ }^{10}$. This highlights the importance of regular engagement in trauma management or re-enforcement of knowledge through re-fresher sessions, to keep knowledge and skills up-to-date.

This review also finds that the PTC course results in positive changes to clinical practice and systems improvement for trauma care. Candidates report intended and actual improvements in their systematic approach to the trauma patient ${ }^{22}$. There are also departmental and institutional benefits, with trauma patients experiencing reduced transfer time to arrival at the emergency department and time to receiving treatment $3^{\circ}$. Some healthcare workers who had not had formal training at a PTC course received in-house training from PTC certified staff ${ }^{22}$. This illustrates their willingness to adopt PTC principles and educate front-line staff, thus ensuring members of local trauma team follow an established protocol to manage trauma in an effective systematic way. PTC, therefore, appears to have a dual benefit to both pre-hospital and facility-based care, promoting key principles set out by the WHO Emergency Care Systems Framework ${ }^{32}$.

There are however suggestions that it is not sufficient to merely 'attend' a PTC course. Cioè-Peña reported that candidates had not effectively adopted PTC principles into their clinical practice during observation of 148 trauma cases ${ }^{24}$. In a follow up study to establish the reasons why, 
several limitations of the study, and important considerations for the effective delivery of this course were revealed ${ }^{33}$. Some candidates were not present for the entirety of the course, having to tend to unwell or private patients. Others were 'mentally absent' as they attended immediately following a night shift, or were distracted by clinical duties 33 . Other issues raised included inadequate preparation time to digest the course reading material, as well as a paucity of essential equipment to appropriately manage trauma patients, such as neck collars ${ }^{33}$. The lack of nurse training as part of trauma courses was also identified as a key barrier, highlighting the importance of medical staff (doctors, nurses and other allied healthcare professionals) to be trained collectively as a team to improve overall trauma care ${ }^{33}$. The authors conclude that institutional, structural and cultural barriers to systems change must be considered alongside any educational intervention to ensure improvements in overall trauma care 33 .

Trauma management courses were introduced to reduce the burden major trauma has on morbidity and mortality. Our review only identified one study that evaluated the effect of widespread PTC course training of front-line workers on mortality after trauma. Although these statistically significant beneficial effects were only demonstrated at one institution, a large number of trauma patients were involved. However, quantifying the magnitude of mortality reduction as a direct result of PTC courses is difficult to ascertain without controlling for numerous other confounding factors. Comparably, despite being the gold standard principle used to manage major trauma in higher income countries, clear evidence demonstrating the beneficial effects of ATLS training on morbidity and mortality is still limited ${ }^{10,14}$.

Although the studies seem to show improvements in knowledge and confidence of participants and a beneficial effect on mortality rates, several limitations exist. Several studies contained within this review assessed knowledge and confidence using immediate post course questions. It 
is therefore difficult to ascertain whether these findings positively correlate with an overall improvement in trauma management and decrease in mortality rates. Further research is required, perhaps using objective measures to establish whether systematic approaches to trauma management using principles learned on the PTC course is being adhered to, similar to the study conducted by Cioè-Peña ${ }^{24}$. In addition, it would be useful to prospectively and objectively report on current systems of trauma management in institutions where front-line staff have not yet received formal trauma management training, prior to introduction of the PTC course.

Limitations may also exist at review level, mainly as a result of incomplete retrieval of published articles. The authors did attempt to minimise this risk by executing the search strategy three times on each specified database. The review limited results to the English language and therefore may have not captured the worldwide data on PTC courses.

\section{Conclusion}

Departmental, institutional and personal improvements may occur in clinical practice as a result of formal PTC training of trauma team members in LMICs. These results support the notion that all front-line staff in LMICs should be offered formal training on the PTC course. Further highquality research is needed to evaluate this course's effects on observed change in clinical practice and patient outcomes. This may require long-term multi-center observational and epidemiological studies to assess improvements in morbidity and mortality.

\section{References}

1. World Health Organisation. Injuries and Violence: The Facts.; 2014. www.who.int/healthinfo/global_burden_disease/projections/en/. Accessed May 21, 2019. 
2. World Bank. World Bank Country and Lending Groups.

https://datahelpdesk.worldbank.org/knowledgebase/articles/906519-world-bank-countryand-lending-groups. Published 2019. Accessed July 27, 2019.

3. Mock C, Joshipura M, Arreola-Risa C, Quansah R. An Estimate of the Number of Lives that Could be Saved through Improvements in Trauma Care Globally. World J Surg. 2012;36(5):959-963. doi:10.1007/soo268-012-1459-6

4. Wisborg T, Montshiwa TR, Mock C. Trauma research in low- and middle-income countries is urgently needed to strengthen the chain of survival. Scand J Trauma Resusc Emerg Med. 2011;19:62. doi:10.1186/1757-7241-19-62

5. Bundu I, Lowsby R, Vandy HP, et al. The burden of trauma presenting to the government referral hospital in Freetown, Sierra Leone: An observational study. African J Emerg Med. 2019;9:S9-S13. doi:10.1016/J.AFJEM.2018.07.008

6. Reynolds TA, Stewart B, Drewett I, et al. The Impact of Trauma Care Systems in Low- and Middle-Income Countries. Annu Rev Public Health. 2017;38(1):507-532.

doi:10.1146/annurev-publhealth-032315-021412

7. Mock C, World Health Organization. Violence and Injury Prevention. Strengthening Care for the Injured[?: Success Stories and Lessons Learned from around the World. World Health Organization; 2010.

https://books.google.co.uk/books?hl=en\&lr=\&id=FdDR887QFeAC\&oi=fnd\&pg=PP2\&ots=v ok67WCtwK\&sig=EUxcjXijkPopsgnWftoAOse77GY\&redir_esc=y\#v=onepage\&q\&f=false. Accessed July 27, 2019.

8. Mock CN, Quansah R, Addae-Mensah L, Donkor P. The development of continuing education for trauma care in an African nation. Injury. 2005;36(6):725-732. doi:10.1016/J.INJURY.2004.12.044

9. Carmont MR. The Advanced Trauma Life Support course: a history of its development and 
review of related literature. Postgrad Med J. 2005;81(952):87-91.

doi:10.1136/pgmj.2004.021543

10. Mohammad A, Branicki F, Abu-Zidan FM. Educational and Clinical Impact of Advanced Trauma Life Support (ATLS) Courses: A Systematic Review. World J Surg. 2014;38(2):322329. doi:10.1007/s00268-013-2294-0

11. Bonnet $\mathrm{E}$, Lechat $\mathrm{L}$, Ridde $\mathrm{V}$. What interventions are required to reduce road traffic injuries in Africa? A scoping review of the literature. Shah TI, ed. PLoS One. 2018;13(11):e0208195. doi:10.1371/journal.pone.0208195

12. Pham H, Puckett $Y$, Dissanaike S. Faster on-scene times associated with decreased mortality in Helicopter Emergency Medical Services (HEMS) transported trauma patients. Trauma Surg Acute Care Open. 2017;2(1):e000122. doi:10.1136/tsaco-2017-000122

13. Moran CG, Lecky F, Bouamra O, et al. Changing the System - Major Trauma Patients and Their Outcomes in the NHS (England) 2008-17. EClinicalMedicine. 2018;2-3:13-21. doi:10.1016/j.eclinm.2018.07.001

14. Jayaraman S, Sethi D. Advanced trauma life support training for hospital staff. In: Sethi D, ed. Cochrane Database of Systematic Reviews. Chichester, UK: John Wiley \& Sons, Ltd; 2009:CDo04173. doi:10.1002/14651858.CDo04173.pub3

15. Nolan JP. Advanced trauma life support in the United Kingdom: time to move on. Emerg Med J. 2005;22(1):3-4. doi:10.1136/emj.2004.018507

16. Petroze RT, Byiringiro JC, Ntakiyiruta G, et al. Can focused trauma education initiatives reduce mortality or improve resource utilization in a low-resource setting? World J Surg. 2015;39(4):926-933. doi:10.1007/s00268-014-2899-y

17. Chokotho $\mathrm{L}$, Jacobsen $\mathrm{KH}$, Burgess $\mathrm{D}$, et al. A review of existing trauma and musculoskeletal impairment (TMSI) care capacity in East, Central, and Southern Africa. Injury. 2016;47(9):1990-1995. doi:10.1016/j.injury.2015.10.036 
18. Primary Trauma Care Foundation. Primary Trauma Care.

https://www.primarytraumacare.org/. Published 2019. Accessed May 21, 2019.

19. Wilkinson D, McDougall R. Primary trauma care. Anaesthesia. 2007;62 Suppl 1(s1):61-64. doi:10.1111/j.1365-2044.2007.05301.x

20. Ley Greaves R, Wilkinson L, Wilkinson D. Primary trauma care: a 20-year review. Trop Doct. 2017;47(4):291-294. doi:10.1177/0049475517704613

21. Peter NA, Pandit H, Le G, Nduhiu M, Moro E, Lavy C. Delivering a sustainable trauma management training programme tailored for low-resource settings in East, Central and Southern African countries using a cascading course model. Injury. 2016;47(5):1128-1134. doi:10.1016/j.injury.2015.11.042

22. Ologunde R, Le G, Turner J, et al. Do trauma courses change practice? A qualitative review of 20 courses in East, Central and Southern Africa. Injury. 2017;48(9):2010-2016. doi:10.1016/j.injury.2017.06.007

23. Sadiq M, Rehman KU, Tariq N, Bashir EA. Impact of Primary Trauma Care Workshop On The Cognitive Domain of Final Year Medical Students. J Surg Pakistan. 2018;23(2):64-67. http://old.jsp.org.pk/lssues/JSP 23 (2) April - June 2018/Muhammad Attiq Sadiq OA.pdf. Accessed May 21, 2019.

24. Cioè-Peña E, Granados J, Wrightsmith L, Henriquez-Vigil A, Moresky R. Development and implementation of a hospital-based trauma response system in an urban hospital in San Salvador, El Salvador. Trauma. 2017;19(2):118-126. doi:10.1177/1460408616672491

25. Thomas BH, Ciliska D, Dobbins M, Micucci S. A Process for Systematically Reviewing the Literature: Providing the Research Evidence for Public Health Nursing Interventions. Worldviews Evidence-Based Nurs. 2004;1(3):176-184. doi:10.1111/j.1524-475X.2004.04006.X

26. Amiri H, Gholipour C, Mokhtarpour M, Shams Vahdati S, Hashemi Aghdam Y, Bakhshayeshi M. Two-day primary trauma care workshop: Early and late evaluation of 
knowledge and practice. Eur J Emerg Med. 2013;20(2):130-132.

doi:10.1097/MEJ.obo13e328356o8c6

27. Nogaro M-C, Pandit H, Peter N, et al. How useful are Primary Trauma Care courses in subSaharan Africa? Injury. 2015;46(7):1293-1298. doi:10.1016/j.injury.2015.04.010

28. Jawaid M, Ahmed Memon A, Masood Z, Nadeem Alam S. Effectiveness of the Primary Trauma Care Course: Is the outcome satisfactory? Pakistan J Med Sci. 2013;29(5):12651268. http://www.ncbi.nlm.nih.gov/pubmed/24353733. Accessed May 21, 2019.

29. Alwawi A, Amro N, Injaya B. The Effectiveness of the Primary Trauma Care Courses in West Bank, Palestine: Are the Outcomes Acceptable? J Educ Pract. 2019;10(9). doi:10.7176/JEP/10-9-12

30. Shi X-P, Qin L-J, Chang Y-X, Li F-L, Wang P. Systemic analysis of pre-hospital trauma emergency treatment in Zhengzhou. J Acute Dis. 2019;8(1):34. doi:10.4103/22216189.250376

31. Cervero RM, Gaines JK. The Impact of CME on Physician Performance and Patient Health Outcomes: An Updated Synthesis of Systematic Reviews. J Contin Educ Health Prof. 2015;35(2):131-138. doi:10.1002/chp. 21290

32. World Health Organisation. WHO Emergency Care System Framework. WHO. https://www.who.int/emergencycare/emergencycare_infographic/en/. Published 2017. Accessed July 28, 2019.

33. Dickason RM, Cioè-Peña E, Chisolm-Straker M. Primary trauma care curriculum: A qualitative analysis of impediments to improvement. Trauma. 2017;19(2):127-132. doi:10.1177/1460408616675641

\section{Figure legends:}

Figure 1 - PRISMA diagram depicting search results. 


\section{Table Legends:}

Table 1 - Inclusion (screening) criteria

Table 2 - Summary of included papers

\section{Supplementary Information:}

Appendix 1: Search Strategy

Appendix 2: Bias assessment of included papers. 